\title{
"Cabergoline, Levosimendan and Iabp: Treatment of Peripartum Cardiomyopathy"
}

\author{
Silvia Donfrancesco ${ }^{\mathrm{a}}$, Marzia Cottini, ${ }^{\mathrm{b}}$, Angela Lappa ${ }^{\mathrm{a}}$, \\ Cecilia Nencini $^{a}$, Patrizia Picozzi ${ }^{b}$, Fabio Sbaraglia ${ }^{b}$, \\ Vincenzo Polizzi ${ }^{b}$, Paola Lilla Della Monica ${ }^{b}$, Agnese Ricotta ${ }^{b}$, \\ Antonio Menichetti ${ }^{a}$, Francesco Musumeci, ${ }^{b}$ \\ ${ }^{\mathrm{a} D e p a r t m e n t}$ of Heart and Vessels, Cardiovascular Intensive Care Care Unit, \\ "S. Camillo-Forlanini” Hospital, 00149 Rome, Italy \\ ${ }^{\mathrm{b}}$ Department of Heart and Vessels, Cardiac Surgery Unit and Heart Transplantation Center, \\ “S. Camillo-Forlanini” Hospital, 00149 Rome, Italy \\ *marzia.cottini@hotmail.it
}

Abstract: PeriPartum CardioMyopathy (PPCM) is a rare but potentially devastating cause of heart failure. We reported the case of a 31-year-old female who developed cardiogenic shock twelve hours post-delivery secondary to PPCM that was successfully treated with combined supportive care, including the cabergoline, levosimendan and IABP.

\section{INTRODUCTION}

PPCM was a form of dilated cardiomyopathy and was diagnosed when there are these three criteria: 1) heart failure develops in the last month of pregnancy or within 5 months of delivery; 2) heart pumping function is reduced, with an left ventricular ejection fraction (LVEF) less than 45\%; 3) no other cause for heart failure $(1,2)$.

PPCM is uncommon disorder associated to dilated cardiomyopathy of unknown origin, that is unique to the pregnant women of all reproductive ages (3). PPCM is rare in the USA, Canada, and Europe, with an estimated case rate of 1 per 2500 to 4000 live births. Most patients (80\%) present within 3 months of delivery, with the minority presenting in the last month of pregnancy $(10 \%)$ or 4 to 5 months postpartum $(10 \%)$ and $(2,4)$. PPCM has a high morbidity and mortality rate ranging from $7 \%$ to $50 \%$ and its outcome is highly variable (5). The basis of human PPCM cannot be explained by a single etiology, thus, the disease has multifactorial origin (2-6). In acute cases, treatment may involve the use of intravenous vasodilatation, inotropic medications, an intra-aortic balloon pump, ventricular-assist devices, and extracorporeal membrane oxygenation. In severe cases, women experience a rapid deterioration in health without improvement even with medical therapy, and may require cardiac transplantation or die of heart failure, thromboembolic events and cardiac arrhythmias.

There is active research into understanding the cause of PPCM and developing new therapies. Treatments that alter the immune system such as intravenous $\gamma$-globulin and immunoabsorption have been tried. Recently, investigators have focused on the role of prolactin in PPCM.

\section{CASE REPort}

A 31-year old female with no past medical history but recent delivery, was referred with progressively worsening dyspnoea and renal failure (anuria) to Emergency room. A Computed Tomography showed no aortic dissection and pulmonary embolism.

Due to congestive heart failure (low oxygen saturation, pulmonary oedema, $\mathrm{PaO} 2 / \mathrm{FiO} 2$ 40, $\mathrm{SpO} 2 / \mathrm{FiO} 2222$, Arterial Blood Pressure [ABP] $80 / 50 \mathrm{mmHg}$, and renal failure), the patient was recovery in our Cardiovascular Intensive Care Unit and required to proceed to tracheal intubation and mechanical ventilation improving high levels of Positive End Expiratory Pressure (PEEP).

A Transthoracic Echocardiography (TTE) was performed and documented dilated and hypokinetic left ventricle (Left Ventricle End-Diastolic Volume [LVEDV] $100 \mathrm{~mL}$, Left Ventricle End Diastolic Diameter [LVEDD] 58 mm, LVEDV/m² 68.03, Cardiac Output [CO] 20 x $120 \mathrm{~L} / \mathrm{min}$, Cardiac Index 
[CI] $1.47 \mathrm{~L} / \mathrm{min} / \mathrm{m}^{2}$ ), Left Ventricle Ejection Fraction (LVEF) of 20\%, Systolic Pulmonary Arterial Pressure (PAPs) $35 \mathrm{mmHg}$, Pulmonary Vascular Resistance (PVR) $3 \mathrm{WU}$, and no valvular dysfunction. The Lab work revealed a troponin-I of $6.02 \mathrm{ng} / \mathrm{ml}$, CPK of $434 \mathrm{IU} / \mathrm{L}, \mathrm{N}$-terminal pro Brain Natriuretic Peptide (NT-proBNP) 1107.4 pg/ml, prolactin (PRL) 192 ng/ml and leukocytosis $31 \times 10^{3} / \mathrm{uL}$.

Following the critical hemodynamic deterioration, the Norepinephrine and Epinephrine infusions were started $(0.1 \mathrm{mcg} / \mathrm{Kg} / \mathrm{min}$ ) and Intra-Aortic Balloon Pump (IABP) was positioned (volume $30 \mathrm{ml}$, 1:1). Methylprednisolone was administered at the dosage of $1 \mathrm{~g} /$ daily for the first three days and 1 $\mathrm{mg} / \mathrm{Kg} /$ day for the following days, in association with Cabergoline $0.5 \mathrm{mg} /$ daily for two consecutive days as a prolactin blocker (Figure 1). Also, Levosimendan (Simdax ${ }^{\circledR}$, Orion Corporation Espoo, Finland) was started with a dose of $0.1 \mathrm{mcg} / \mathrm{Kg} / \mathrm{min}$ over the 24 hours. The cardiac and respiratory assessments were stabilized and the diuresis re-started (about $50 \mathrm{ml} / \mathrm{h}$ ).<smiles>C=CCN1C[C@H](C(=O)N(CCCN(C)C)C(=O)NCC)C[C@H]2c3cccc4[nH]cc(c34)C[C@H]21</smiles>

Figure1. A sharp drop in prolactin and NT-proBNP levels is observed after cabergoline administration.

A control TTE was performed after the finished- Levosimendan therapy and showed an improvement of LVEF (30\%), moderate hypokinesis and reduction of LV end-diastolic pressure ( $<20 \mathrm{mmHg}$ ).

Afterwards, we optimized the inotropic drugs by switching the Epinephrine with Dobutamine (4 $\mathrm{mcg} / \mathrm{Kg} / \mathrm{min})$ and decreasing Norepinephrine infusion $(0.05 \mathrm{mcg} / \mathrm{Kg} / \mathrm{min})$ in order to increase the positive cardiac effect of $B 1$ and $B 2$ receptor stimulations. No $\beta$-blockers was administrated to the patient, Ivabradine $(5 \mathrm{mg} / 12 \mathrm{~h}$ ) was used to control sinus tachycardia (median HR $120 \mathrm{bpm}$ ). The Cabergoline was continued and the control PRL was $9.7 \mathrm{ng} / \mathrm{ml}$ (Table 1).

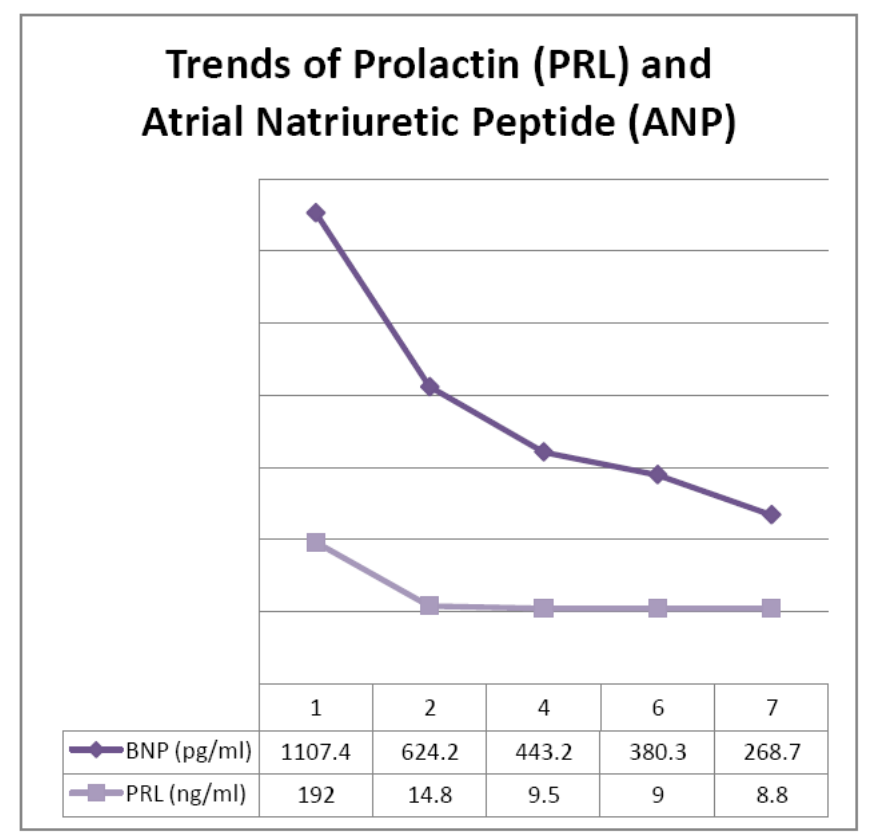


On the fifth post-recovery day, the patient was weaned from mechanical ventilation, a second dose of Levosimendan was administered to help weaning from IABP. The patient was discharged with a mild dose of ACE-inhibitor and B-blocker on the 21st post-recovery day. The discharged TTE documented the cardiac functional recovery of contractility, LVEF 52\% (Table 2).

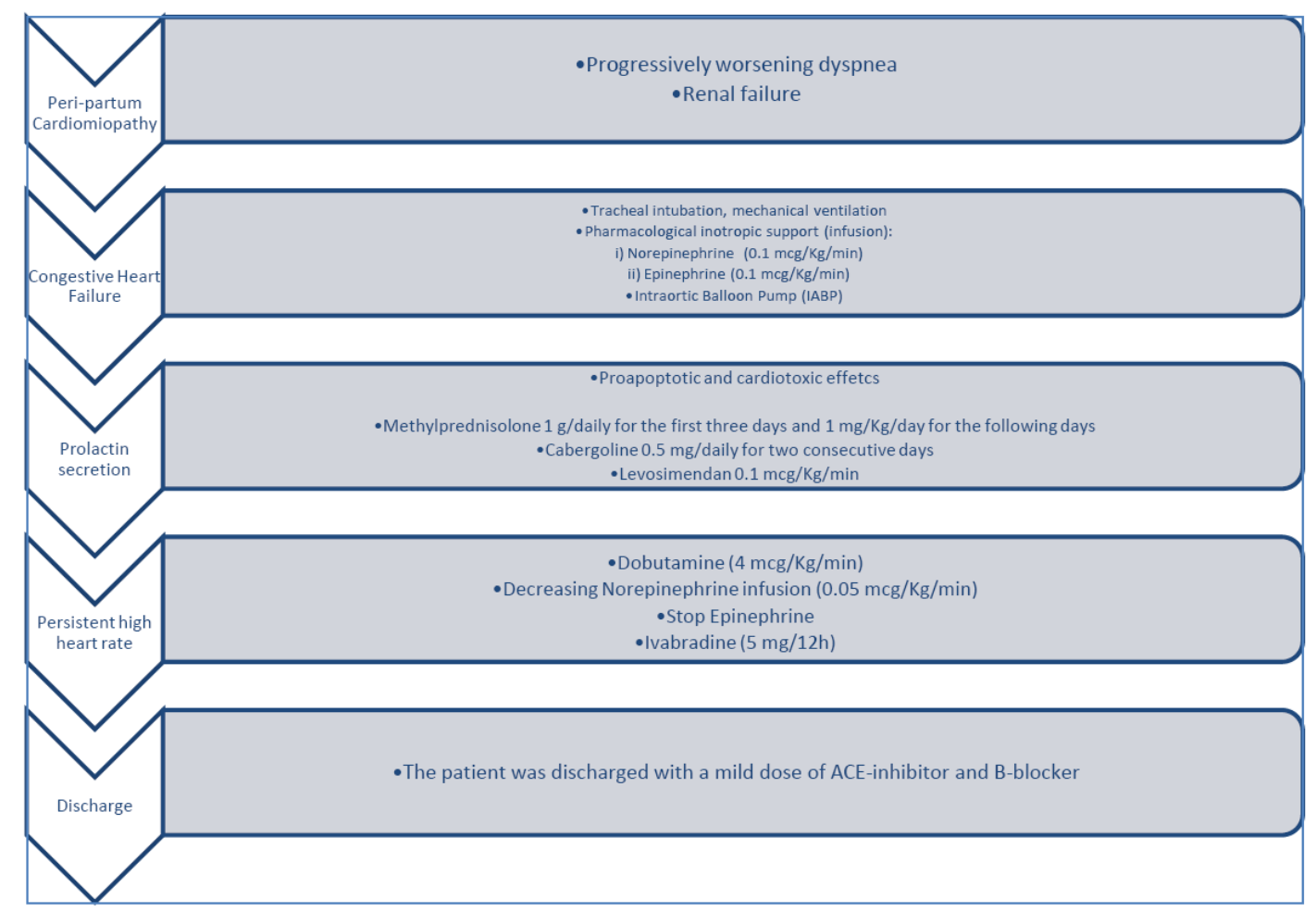

\section{DISCUSSION}

PPCM is uncommon disorder associated to dilated cardiomyopathy of unknown origin, that is unique to the pregnant women of all reproductive ages (1-6). Reported incidences for PPCM vary among different geographic regions with potential hotspots in Africa (1:100 to 1: 1000) and Haiti (1:299). In Western societies, the incidence of PPCM is rising (in the USA from 1 in 4350 in 1993 to 1 in 2229 in 2002) possibly because of socio-economic changes (rising maternal age, fertility assisted treatments, and multifoetal pregnancies), better diagnostic tools and increasing awareness created by large prospective international registry (8-7).

Current advances in knowledge on pathomechanisms of PPCM documented a potential role of low selenium levels, various viral infections, stress-activated cytokines, inflammation, autoimmune reaction, a pathological response to haemodynamic stress, and unbalanced oxidative stress. A novel finding is the discovery that oxidative stress-mediated cleavage of the nursing hormone prolactin into a smaller biologically active subfragment, $16-\mathrm{kDa}$ prolactin, may be a major factor initiating and driving PPCM. This 16-kDa prolactin up-regulates miR-146a, which mediates most of the adverse effects of 16-kDa prolactin in endothelial cells and is released in microparticles (exosomes) into the circulation from where it also affects cardiomyocytes. Along this line, multiple anti-angiogenic factors such as 16-kDa prolactin and sFlt1 disturb the angiogenic balance in the peripartum phase thereby causing vascular impairment and subsequently heart failure (1-7).

According to the ESC guidelines of heart failure (4) and ACCF/AHA guideline for management of heart failure (5), PCCM therapy could be treated by standard therapy for heart failure including betablockers, ACE-inhibitors/AT1-blockers, mineralocorticoid receptor antagonists (MRA), and diuretics after delivery. As no data on the use of inotropes exist in patients with PPCM and catecholamines may aggravate myocardial damage, an i.v. infusion of an inotrope should only be considered in patients with severe hypotension and/or signs of cardiogenic shock. As soon as haemodynamic stability is achieved, inotropes should be tapered and general recommendations for patients with heart failure in pregnancy should be followed. Early treatment with beta-blockers started at very low dosages appears to be protective even in patients with severely depressed ejection fraction. In patients with persistent sinus tachycardia, in whom uptitration of beta-blocker dose was not feasible due to hypotension or heart failure, we also have good experience with the use of Ivabradine (10). 
Cabergoline was ergoline derivative like Bromocriptine and dopamin agonist which decreased the level of prolactin. It had an extremely long half-life (65 hours) and was highly selective for dopamine D2-receptor. Despite several reports had documented the potential efficacy of Bromocriptine in PPCM, to the best of our knowledge there is only few other published case reports that describes the use of Cabergoline $(6,7)$.

In the scientific literature, the increase of cabergoline in PPCM was described by Scardovi et al (11), Mahmod et al (12), and John et al (13). It documented an improvement in PPCM care patient.

Our patient satisfied all current criteria for PPCM: the onset of the disease occurred twelve hours after delivery; no other identifiable causes of heart failure were present; no cardiac diseases prior to the last month of pregnancy were reported and the patient presented left ventricular dysfunction (LVEF 20\%). An early first cycle of Levosimendan was administered due to its ability to improve cardiac performance by optimising the response of contractile proteins to calcium without increasing its intracellular levels, unlike traditional inotropes which increase myocardial oxygen demand and the risk of arrhythmia $(8,9)$.

According to our experience, the Levosimendan and Carbergoline could be the optimal therapeutic combination in optimal cardiac functional recovery. It was documented by the trend of BNP and PRL.

Moreover, the persistent sinus tachycardia was treated with ivabradine $(6,7)$. This new HR reducing agent acted on the sino-atrial node (SA) through a selective inhibition of the pacemaker $\mathrm{I}_{\mathrm{f}}$ current in a dose-dependent way. Ivabradine can slow the HR without a reduction of cardiac output in both patients with advanced HF and in those with chronic stable angina when beta blockers are contraindicated.

In case of PPCM, we suggested a multimodal therapeutic approach in case of cardiogenic shock. We reported a successful treatment involving either pharmacological or mechanical supports: the IABP and the novel combination of drugs (Cabergoline, Ivabradine, Levosimendan) had rebalanced the cardiac performance optimizing gradually cardiac output, cardiac input, heart rate, stroke volume, level of PRL.

PPCM is a rare but life-threatening disease. In recent years, many efforts have done to increase knowledges and therapeutic options to PPCM treatments. Cabergoline is a potent dopamine receptor agonist and which decreased the level of prolactin. The combination of cabergoline, ivabradine and levosimendan may improve the cardiac performance in PPCM.

Optimal timing of diagnosis in PPCM and multimodal therapy could reduce the morbidity and mortality of PPCM but more clinical data and could be collected and analysed to confirm our experience.

Human rights statements and informed consent: All procedures followed were in accordance with the ethical standards of the responsible committee on human experimentation (institutional and national) and with the Helsinki Declaration of 1964 and later revisions. Informed consent was obtained the patietn for being included in the case report (verbal and written format), also we obtained Ethics Committee permission to publish the report.

\section{REFERENCES}

[1] Sliwa K, Hilfiker-Kleiner D, Petrie MC, Mebazaa A et al. Current state of knowledge on aetiology, diagnosis, management and therapy of peripartum cardiomyopathy: a position statement from the Heart Failure Association of the European Society of Cardiology Working Group on peripartum cardiomyopathy. Eur J Heart Fail 2010; 12: 767-78.

[2] Givertz MM. Peripartum Cardiomyopathy. Circulation 2013; 127: e622-e626.

[3] Johnson-Coyle L, Jensen L, Sobey A. American College of Cardiology Foundation, American Heart Association. Peripartum cardiomyopathy: Review and practice guidelines. Am J Crit Care. 2012;21:89-98.

[4] Regitz-Zagrosek V, et al. ESC Guidelines on the management of cardiovascular diseases during pregnancy: the Task Force on the Management of Cardiovascular Diseases during Pregnancy of the European Society of Cardiology (ESC). Eur Heart J 2011;32:3147 -3197. 
[5] Clyde W. Yancy, et al. 2013 ACCF/AHA Guideline for the Management of Heart FailureA Report of the American College of Cardiology Foundation/American Heart Association Task Force on Practice Guidelines. J Am Coll Cardiol. 2013;62(16):e147-e239. doi:10.1016/j.jacc. 2013.05.019.

[6] De Jong JSSG, Rietveld K, van Lochem LT, Bouma BJ. Rapid left ventricular recovery after cabergoline treatment in a patient with peripartum cardiomyopathy. Eur J Heart Fail 2009; 11:220-2. (doi: 10.1093/eurjhf/hfn034)

[7] Masliza Mahmod, Cheuk F Chan, Aamir Ali, Sadaf Raza, Nik R Wan-Ibrahim, Georgios Manolis, Rahana Abd Rahman, Ankur Gulati. Cabergoline as an adjunct for early left ventricular recovery in peripartum cardiomyopathy. Br J Cardiol 2011;18:243-245doi:10.5837/bjc.2011. 008.

[8] Nquyen HD, McKeown B. Levosimendan for post-partum cardiomyopathy. Critical Care Resusc 2005;7:107-10.

[9] Adamopoulos S, Parissis JT, Lliodromitis EK et al. Effects of levosimendan versus dobutamine on inflammatory and apoptotic pathways in acutely decompensated chronic heart failure. Am J Cardiol 2006; 98:102-6.

[10] De Ferrari GM, Mazzuero A, Agnesina L, Bertoletti A et al. Favourable effects on heart rate reduction with intravenous administration of ivabradine in patients with advanced heart failure, Eur J Heart Fail 2008; 10: 550-5.

[11] Scardovi AB, De Maria R, Ricci R. Acute peripartum cardiomyopathy rapidly evolving in cardiogenic shock. Int J Cardiol 2015;189:255-6.

[12] Mahmod M, et al. Cabergoline as an adjunct for early left ventricular recovery in peripartum cardiomyopathy. Br J Cardiol 2011;18:243-245.

[13] Jong JS, et al. Rapid left ventricular recovery after cabergoline treatment in a patient with peripartum cardiomyopathy. Eur J Heart Fail 2009;11:220-2. 